\title{
Ein durch Resektion geheilter Fall von Gangrän des Dickdarms im Gebiete der A. mesenterica inferior nebst einem Verfahren die Kontinuität des Darms durch ein Stück Ileum wiederherzustellen.
}

Von Dr. R. Faltin. Dozent für Chirurgie an der Universität Helsingfors (Finnland).

(Mit 2 Abbildungen.)

Folgenden Fall habe ich die Gclegenheit gehabt in der chirurgischen Universitätsklinik zu behandeln:

J. N., Buchhalter, 33 Jahre alt, aus Helsingfors. Aufgenommen am Abend des 28. X. Igro.

Keine hereditäre Belastung. Als Kind gesund. Vor 9 Jahren Typhus. War 6 Wochen bettlägerig. Eine Zeit lang später Diarrhöe mit 2-3 Stühlen täglich. In den letzten 6 Jahren sind dagegen die Darmfunktionen äußerst träge gewesen, so daß er meistens gezwungen gewesen ist, jeden zweiten oder dritten Tag ein ca. ${ }^{3 / 4}$ Liter großes Wasserklistier zu nehmen. Vor ca. 3 Jahren litt er während einiger Tage an unbestimmten Bauchschmerzen mit Verstopfung. Im Frühjahr IgIo erkrankte er plötzlich mit heftigen Bauchschmerzen und blutigen Diarrhöen, welche 4 Tage anhielten. Wurde nach einem Krankenhause gebracht, wo man - nach gütiger Mitteilung des behandelnden Arztes eine akute Colitis annahm und ihn 2 Wochen dafür behandelte. In den letzten 2 Jahren mitunter an gelinden Schmerzen in den Schultergegenden gelitten.

Am 28. X., als der Patient sich gerade eins von seinen gewöhnlichen Wasserklistieren applizieren ließ, weil er in den letzten Io Tagen keinen ordentlichen Stuhlgang gehabt hatte - nur einzelne kleine schleimbedeckte Fäzesballen waren abgegangen, bekam er außerordentlich heftige Schmerzen im Bauch, so daß die Verabreichung des Klistiers abgebrochen werden mußte. Das Wasser floß gleich wieder heraus und war mit etwas Blut vermengt. Ein hinzugerufener Arzt verordnete eine Arznei, die sofort erbrochen wurde. Während des Tages heftige Bauchschmerzen. Totale Verhaltung von Stuhl und Winden.

S t a t us bei der Aufnahme: Gut genährter Mann von gewöhnlichem Körperbau. Stark angegriffen. Gesichtsfarbe bleich. Zunge rein. Temperatur 37,8 bwz. 38,7 im Rektum. Puls regelmäßig, klein, Ioo. Herz von gewöhnlicher Größe. Töne rein und klar. Harn klar, spez. 
Gew. Iozo ohne abnorme Bestandteile. Klagt über heftige, anhaltende Bauchschmerzen. Bauch etwas aufgetrieben, ohne lokalen Meteorismus. Bauchdecken gespannt. Überall Druckempfindlichkeit, aber besonders im Verlauf des Colon descendens und in der linken Fossa iliaca. Per rectum nichts Abnormes. Leberdämpfung erhalten.

Um 9 Uhr abends $L$ a par o to mie. Schnitt in der Linea alba. Trübe fäkalstinkende Flüssigkeit entleert sich. Die Dünndarmschlingen zusammengefallen, injiziert. Die Flexura sigmoidea von gewöhnlicher Größe und Lage, weder torquiert noch irgendwie disloziert, nicht meteoristisch, aber vollkommen gangränös. Die Gangrän erstreckt sich aufwärts über das ganze Colon descendens, inklusive Flexura coli lienalis, und über eine handbreite, medialwärts ziemlich scharf begrenzte Partie des Quercolons. Der gangränöse Darm ist vollkommen schlaff und mit ausgebreiteten Hämorrhagien besetzt. Stellenweise finden sich Flecke von scheinbar normaler Beschaffenheit und mit spiegelnder Serosa. Abwärts nimmt die Mißfärbung der Darmwand etwas $a b$, so daß im Colon pelvinum und im oberen Teil der Pars pelvina recti nur einzelne Hämorrhagien zu sehen sind, während der unterste Teil derselben normal erscheint.

Der gangränöse Darm wird von seinem Mesenterium gelöst und durch eine etwa in Nabelhöhe am Rande des linken M. rectus angelegte Wunde herausgezogen, was ohne besondere Spannung geschehen kann. Nach Befestigung der zu- und der abführenden Darmteile, d. h. des Quercolon und des Colon pelvinum in paralleler Stellung aneinander und in die Wunde, wird der gangränöse Darm abgetragen. Die große mediane Bauchwunde wird exakt vernäht. Ein Jodoformgazetampon kommt ins kleine Becken neben dem Rektumstumpf.

Beschreibung des entfernten gangränösen Darmes. Das Darmstück ist nach Aufbewahrung in Formalinlösung in geschrumpftem Zustande ca. $45 \mathrm{~cm}$ lang. Seine äußere Fläche ist dunkel und mit Hämorrhagien besetzt. Nur im mittleren Teil des Colon descendens liegt eine etwa $I_{5} \mathrm{~cm}$ lange Partie, wo der Darm fleckenweise, namentlich am Mesenterialansatz, 'ein ziemlich normales Aussehen zeigt. Beim Aufschneiden des Darms ist die diesen Stellen entsprechende Schleimhaut stark geschwollen und von fleckigem Aussehen, in dem kleine hellere Flecke mit vollkommen brandigen, großen, schwarzen abwechseln. An allen übrigen Stellen ist der entfernte Darm in seiner. ganzen Dicke vollkommen gangränös. Keine Fäzes im Lumen des Darmes.

29. X. Magenspülungen am Morgen und am Abend. Große Mengen gallenfarbiger Flüssigkeit. Intravenöse Kochsalzinfusion. 2. XI. Behandlung täglich wie oben, weil sonst kopiöses Erbrechen. Durch den Anus praeternaturalis werden sehr große Fäzesmassen herausgespült. Wegen stetig steigender Temperatur Revision der Wunde und Entfernen einiger Nähte. Am Abend entdeckte die Wärterin, daß eine ca. $15 \mathrm{~cm}$ lange Dünndarmschlinge durch die mediane 
Wunde prolabiert hatte. Reposition derselben in Narkose. - Bis zum 7. XI. tägliche Magenausspülungen immer nach I2 Stunden, weil der Magen sich nach dieser Zeit stets übervoll zeigte und Patient starkes Unbehagen empfand. Flüssigkeit wurde dem Patienten außer per os mit Tropflavements in den zuführenden Darm verabreicht. Der abführende Darm gangränesziert. - 9. XI.: Keine Magenausspülungen seit dem 7. nötig gewesen. Beim Ziehen am gangränösen Rektumstumpf folgt ein ca. Io $\mathrm{cm}$ langes, vollkommen abgestorbenes Stück. Ein Drain wird eingeführt. Per rectum kann man das untere Ende desselben in der Ampulla recti palpieren. - Patient erholte sich allmählich. Durch tägliche und reichliche Darmspülungen konnten ausgiebige Entleerungen erzielt werden. In der Zwischenzeit entleerte sich nichts durch den Kunstafter. (Patient lag deshalb vollkommen trocken und seine Pflege wurde durch diese Maßnahme ungemein erleichtert.) Beim Touchieren per rectum kann man nicht die obere Grenze des noch erhaltenen Teiles des Rektums mit dem Finger erreichen.

Da Patient darauf drängte baldigst von seinem Anus praeternaturalis befreit $\mathrm{zu}$ werden, wurde am 29. XII. zu einer erneuten Operation geschritten. Zuerst wurde der Stumpf des Colon transversum von der Bauchwand abgelöst. Danach Laparotomie in der Linea alba. Trendelburgsche Lage. Adhärente Dünndarmschlingen werden abgelöst und durch große Kompressen noch oben gedrängt.

Der abführende Dickdarmschenkel ist in seinem oberen Teil zu einem harten Strang eingeschrumpft und in Adhärenzen eingebettet. Er wird abgelöst und durch den After ausgestiilpt, was nur unter großen Schwierigkeiten geschehen kann. Es zeigt sich nun, daß sein oberer Teil, d. h. das ganze Colon pelvinum und der größte Teil der Pars pelvina recti ohne Schleimhaut und ohne Lumen sind und nur aus Serosa und aus Resten der Muscularis bestehen. Er wird deshalb in gesundem abgetragen. Der Rest zieht sich durch den After wieder zurück. Vom Rektum bleiben also bestehen Pars perinealis und ein kleines Stückchen von Pars pelvina.

Es war die Absicht, den mobilisierten Colon transversum-Stumpf ebenfalls durch den After herauszuziehen, um auf diese Weise eine zuverlässige Darmnaht zwischen dem Quercolon und dem Rektumstumpf anlegen zu können. Es war aber nicht möglich den Quercolonstumpf durchzuziehen. Ja, es gelang sogar nur unter bedeutender Spannung, die Darmstümpfe im kleinen Becken einander zu nähern. Da eine Darmnaht zwischen dem gleich oberhalb der Douglasschen Falte abgetragenen Rektum und dem Colon transversum unter diesen Umständen unausführbar erschien, wurde beschlossen die Kontinuität des Darmes durch ein zwischengeschaltetes Ileumstück wiederherzustellen.

$\mathrm{Zu}$ dem $Z$ weck werden die untersten Ileumschlingen hervorgezogen. Siesind zusammengefallen, fast atrophisch und mit ungewöhnlich kurzem 
Mesenterium versehen, so daß keine der Schlingen ohne jede Spannung mit seiner Kuppe an den Rektumstumpf heranreicht. Eine etwa $20 \mathrm{~cm}$ lange, etwa ebenso viele Zentimeter vom Cöcum entfernte Ileumschlinge wird an beiden Enden quer durchschnitten und durch zwei nach der Radix konvergierende ca. $6 \mathrm{~cm}$ lange Schnitte im Mesenterium mobilisiert. Durch eine gewöhnliche zirkuläre Darmnaht mit feiner Seide wird die Kontinuität des Darmes wieder hergestellt. Mit zwei an dem peripheren Ende der ausgeschalteten Ileumschlinge befestigten Seidenfäden wird dieselbe durch das Rektum herabgezogen. Infolge der Spannung des Mesenteriums gelingt dies erst, nachdem dasselbe ca. $2 \mathrm{~cm}$ oberhalb des Ansatzes am Darm quer bis zum gut sichtbaren Hauptgefäß einge-

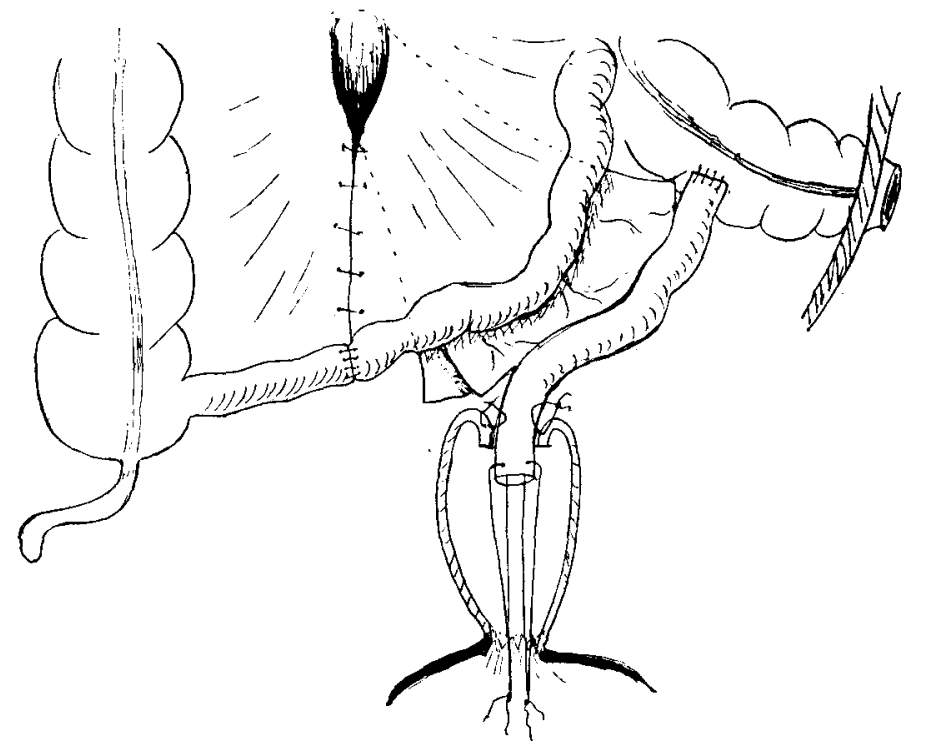

Fig. I.

kerbt worden ist. Auch dann ist die Spannung so groß, daß die Ausführung der Darmnaht außerhalb des Anus, wie anfangs beabsichtigt war, nicht realisierbar erscheint. Um die Spannung aufzuheben, muß man den Ileumstumpf sich wieder ein Stückchen nach oben retrahieren lassen und in dieser Lage durch einige an die Analhaut lose geknotete Nähte provisorisch fixieren. Von der Bauchhöhle aus werden dann nicht ohne Schwierigkeiten einige Knopfnähte angelegt, wodurch eine gewisse Einstülpung des Rektumstumpfes und eine Adaptation der äußeren Fläche desselben gegen die Serosafläche des Ileumstückes erreicht wird. Nach beendeter Sutur ragt das Ileumstück ca. $4 \mathrm{~cm}$ lang portioartig in die Ampulla recti hinein (Fig. I). Das proximale Ende der ausgeschalteten Ileumschlinge wird mit dem Quercolon durch eine etwa 
$6 \mathrm{~cm}$ von dessen freien Ende entfernte End-to-side-Anastomose vereinigt. Dieses wird als Anus praeternaturalis sicherheitshalber an seinen alten Platz befestigt. Schluß der medianen Bauchwunde. Jodoformgazetamponade gegen und um das Ileumstück durch die seitliche Wunde. Fig. I zeigt schematisch wie die Verhältnisse sich nach vollendeter Operation gestalteten.

Nach der langen Operation war Patient anfangs sehr mitgenommen, aber nach einer intravenösen Kochsalzinfusion erholte er sich rasch.

Weiterer Verlauf. Von der tamponierten Wunde entleerte sich anfangs reichlich Eiter. Die das ausgeschaltete Ileumstück nach unten in der Rektalampulle anziehenden Nähte schnitten bald durch und der Darm retrahierte sich nach oben. Doch konnte das Ende leicht mit dem Finger erreicht werden. Täglich wurde der Anus mit den Fingern vorsichtig dilatiert. Hierbei entleerte sich immer reichlich stinkender Eiter. Patient fing an über Tenesmen zu klagen. Nach täglichen vorsichtigen Spülungen besserte sich der Zustand allmählich. Jeden zweiten Tag wurde eine Darmspülung durch den Anus praeternaturalis gemacht. Dabei floß etwas Spülwasser in die Rektalampulle.

Anfangs Februar stand Patient auf. Sowohl spontan als nach den Darmspülungen fingen immer größere Fäzesmengen an per anum abzugehen. Das portioartig sich anfühlende, in die Rektalampulle hineinragende Ileumstück schrumpfte allmählich. Es gelang doch das Lumen digital zu erreichen und $\mathrm{zu}$ erweitern.

Wurde am I5. II. IgII nach Hause entlassen. Wenn der Anus praeternaturalis durch Watte verstopft war, ging der größte Teil der Fäzes, mitunter sogar alles per vias naturales ab. Täglich mehrere, 4-6, weiche Stühle mit etwas Tenesmen verbunden. Später stellte sich Patient vor, immer nach $\mathrm{I}-2$ Wochen. Es konnten bisweilen Wochen vergehen, ohne daß etwas durch den Anus praeternaturalis sich entleerte. Das untere Ileumende hatte sich allmählich so erweitert, daß es am 29. III. für 2 Finger gut passierbar war. Die Stühle wurden allmählich' fester und von ziemlich normaler Form, doch bestand eine gewisse Neigung zu Tenesmen und zu Diarrhöen. Vom Ende April bis Ende Mai waren die Diarrhöen besonders hartnäckig, sogar 9 Stühle täglich, wobei sich mitunter etwas durch den Anus praeternaturalis entleerte. Nach großen Rektalklistieren und Bismuth + Benzonaphtol besserte sich der Zustand, so daß Patient anfangs Juni, als er auf 2 Monate nach seiner russischen Heimat fuhr, sich sehr erholt hatte, und der Darm sich regelmäßig ohne Beschwerden per vias naturales entleerte. Durch den Anus praeternaturalis kam, wenn derselbe durch etwas, Watte zugestopft war, nichts, so daß Patient seinen Dienst als Schreiber anstandslos hatte besorgen können.

Am I6. VIII. wurde Patient von neuem in die Klinik aufgenommen. Er hatte sich sehr erholt. Stuhlgang war regelmäßig gewesen, meistens 3-5 weiche Stühle täglich. Durch den Anus praeternaturalis 
hatte sich hin und wieder etwas entleert. Per rectum konnte das untere, etwas evertierte Ende des Ileumstücks als für 2 Finger gut durchgängig palpiert werden.

Am I7. VIII. wurde der Anus praeternaturalis nach Loslösung von der Bauchwand und Einstülpung geschlossen. Seit dem geht es dem Patienten gut. Die Stühle sind meistens weich, aber auch bisweilen von festerer Konsistenz und dann von normaler Form.

Aus der mitgeteilten Krankengeschichte scheint mir hervorzugehen, daß ein Ileumstück die Funktion eines Colonstückes übernehmen kann. Die anfangs beobachteten Diarrhöen und Tenesmen beruhten wahrscheinlich auf der Reizung der Schleimhaut des Ileumstückes durch die Fäzesmassen. Es scheint aber, als ob sich dieselbe mit der Zeit verloren hätte und das Ileumstück sich an seine neue Funktion gewöhnt hätte. Es besteht nur noch eine gewisse Neigung zu häufigen Stuhlgang, 2-4 mal täglich.

In bezug auf die Diagnose ist der Fall auch epikritisch nicht ganz leicht zu klassifizieren. Am nächsten liegt die Annahme einer Embolie der Arterie, was auch mit dem stürmischen Einsetzen und den übrigen Symptomen der Krankheit am besten harmoniert. Irgendwelcher Ausgangspunkt für eine Embolie war doch nicht ausfindig zu machen. Gegen die Annahme einer Thrombose spricht der Mangel an Arteriosklerose, ohne welche eine primäre Thrombose der Arterie wohl kaum denkbar wäre (W i l m s). Dagegen kann die Möglichkeit einer irgendwie durch die langwierige Verstopfung hervorgerufenen Thrombose der Mesenterialvenen nicht ganz von der Hand gewiesen werden, obgleich es wahrscheinlich ist, daß die Symptome dann nicht so stürmisch gewesen wären. Doch scheinen mir die klinischen Krankheitsbilder, wic auch Wil $\mathrm{m}$ s hervorhebt, nicht so scharf voneinander abzuweichen, daß eine Unterscheidung immer möglich wäre zwischen Embolie und Thrombose, ebenso wenig wie zwischen den S p r e n gelschen Formen der hämorrhagischen Infarzierung und der anämischen Gangrän.

Ich will mich deswegen damit begnügen, festzustellen, daß es sich in meinem Falle um eine zu Gangrän führende Zirkulationsstörung im Gebiete der Arteria mesenterica inferior handelte.

Während Embolien bzw. Thrombosen im Bereiche der Arteria mesenterica sup. verhäItnismäßig häufig beobachtet worden sind, 
scheinen ähnliche Affektionen im Bereiche der Arteria mesenterica inferior sehr seltene Vorkommnisse zu sein, wie aus den Arbeiten von $\mathrm{Deckart}$, Neutra, Brunner, Kölbing, Kös t e r u. a. hervorgeht. Alle zu dieser zweiten Gruppe gehörenden Patienten sind übrigens gestorben.

Etwas günstiger gestaltet sich die Prognose für die zur ersten Gruppe gehörenden Patienten. Sogar Spontanheilungen sollen vorkommen. $\mathrm{N}$ e utra erwähnt I2 solche Fälle. K $\mathrm{K} 1 \mathrm{~b}$ ing berichtet über 8 und $\mathrm{D}$ e $\mathrm{ck}$ art über 6 Heilungen. Doch scheint die Diagnose in der Mehrzahl der Fälle anfechtbar zu sein.

Nach Operationen sind, soweit ich habe finden können, nur die Patienten von E 11 i o t, S p r eng e l, Kölbing, Brun$\mathrm{n}$ e $\mathrm{r}$ und $\mathrm{H}$ a a $\mathrm{g} \mathbf{n}$ mit dem Leben durchgekommen. Hierzu käme nun als sechster mein Patient, der aber in der Beziehung einzig wäre, daß bei ihm die Gangrän den Bereich der Arteria mesenterica inferior betraf.

Diese Daten haben aber hauptsächlich ein Kuriositätsinteresse. Von viel größerem und von prinzipiellem Interesse ist dagegen das von mir gebrauchte Verfahren, um den Dickdarmdefekt durch ein Stück Ileum zu ersetzen.

Als ich bei der Operation vor der Aufgabe, die Kontinuität des Darmes wiederherzustellen, stand, waren mir diesbezügliche frühere Versuche nicht bekannt.

K r o gi us' Verfahren ${ }^{1}$ ) war damals noch nicht veröffentlicht. Dasselbe steht dem meinigen recht nahe, da ja ebenfalls ein Stück Dünndarm benutzt wird, mit der Abweichung doch, daß die Kuppe einer ganzen Schlinge in die Analportion eingefügt wird und nicht das Ende der ausgereckten Schlinge.

Vignolo hat an Hunden den nach der Exstirpation des Rektums und der Flexur entstandenen Defekt durch ein Stück Ileum in der Weise ersetzt, daß nach Herstellung der Kontinuität des Dünndarms die Enden der Schlinge an den Colon- und an den Rektumstumpf durch eine ,Ileo-colostomia latero-lateralis“" bzw. eine "Ileo-rectostomia termino-obliqua-lateralis" befestigt wurden. Um beim Menschen die Kontinuität zwischen Colon-

I) ... oder richtiger Vorschlag, da es bis jetzt am Lebenden nicht ausgeführt worden ist... 
stumpf und Sphinktergegend, z. B. nach der abdomino-perincalen Exstirpation des Rektums, herstellen zu können, schlägt Vignolo vor, dic Dünndarmschlinge in der Art zu mobilisieren, daß das Mesentcrium oberhalb der anastomotischen Bögen in querer Richtung auf ciner Strecke von 8-ro $\mathrm{cm}$ incidiert wird. Die Mobilisierung soll noch besser gelingen, ,wenn zwischen den Gefäßschlingen Incisionen in das Mesenterium ausgeführt werden“" (Gian is Referat). Wic ersichtlich, ist der Gedankengang bei Vignolo dem meinigen sehr ähnlich gewesen. Auch er hat sich die Möglichkeit gedacht, einen durch direkte Vereinigung der Stümpfe nicht aufhebbaren Defekt des Dickdarms durch ein Stück Ileum zu ersetzen.

In anderer Weise ist M o n t p r o f it vorgegangen, um das Problem zu lösen. Den nach Rescktion der Flexur und eines Teiles des Colon descendens entstandenen Defekt hat er ganz cinfach bestehen lassen. Die Stümpfe hat er blind geschlossen, das Ileum nahe am Cöcum abgeschnitten und ins Rektum implanticrt und schließlich das periphere Ilcumstück an das proximale anastomosiert. Dadurch hat er den ganzen Dickdarm, d. h. Colon ascendens, transversum und descendens, ausgeschaltet, was mir doch ein nicht zu empfehlendes Verfahren zu sein scheint. Es kann nicht gleichgültig für den Organismus sein, ob der Dünndarminhalt direkt ins Rektum geleitet wird oder erst den Dickdarm, wo die Resorption namentlich von Flüssigkeiten sehr lebhaft ist, passieren muß. Im ersteren Falle würden wahrscheinlich beständige Diarrhöen sich cinstellen und außerdem wären Komplikationen von dem großen ausgeschalteten Darm nicht ausgcschlossen.

Auf diese Nachteile der einseitigen Ausschaltung des Dickdarms hat neucrdings auch de $Q$ u e r va in hingewiesen. Viel einfacher und natürlicher wäre es, in ähnlichen Fällen den Dickdarm zu mobilisieren und ihn mit dem erhaltenen Tcile des Rektums zu vereinigen. $\mathrm{K}$ ü $\mathrm{m} \mathrm{mell}$ hat ja gezeigt, daß das Colon descendens und transversum durch Loslösung ihrer Mesentericn so ausgiebig mobilisiert werden können, daß ein Einnähen des Stumpfes in den Sphincter ani ohne Spannung möglich wird. Eine Gangrän ist hicrbei im Gegensatz zu den Verhältnissen nach Ablösung des Mesenteriums von der Flexur nicht zu befürchten, 
wenn man sich mindestens I cm vom Darm entfernt hält, um nicht dic Randarteric zu verletzen, denn diese Randarterie ist es, welche nach Durchschneidung der Arteria colica sin. den Darm mit Blut aus der Arteria colica media versorgt.

In meinem Falle war ein Einnähen des Colonstumpfes in den Anus nicht möglich, weil ein zu großer Teil des Quercolons hätte entfernt wcrden müssen. Schon das Einnähen des Colonstumpfes als einen Anus iliacus, obwohl es ohne besondere Spannung hätte ausgeführt werden können, war doch wahrscheinlich nicht ohne Einfluß auf das nach der ersten Operation ungewöhnlich lange anhaltende Erbrechen gewesen. Vielleicht war es der auf den Magen ausgeübte $Z u g$ des eingenähten Quercolons, welcher zum Teil die Entleerung des Magens verhinderte.

Der Fall bot noch ein zweites technisches Problem dar, welches nicht allzu selten dem Chirurgen sich präsentiert, nämlich dic Darmnaht tief im kleinen Becken. Es kann, wie in meinem Falle, vorkommen, daß die Hoheneggsche Umstülpungsmethode nicht gelingt. Würde man dann das Steißbein exstirpieren und das obere Ende des Rektumstumpfes von hinten freilegen, wäre es vielleicht in einigen Fällen möglich, die Naht aus-

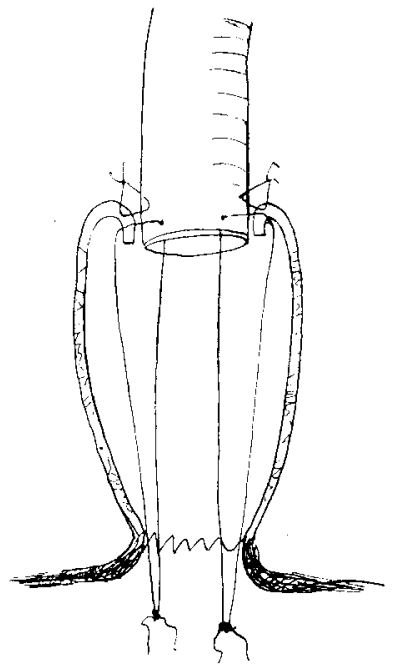

Fig. 2. zuführen. Man wird sich wohl doch selten zur Exstirpation des SteiBbeines nur der Naht wegen entschließen können.

Wenn es aber nur gelingt, den scrosabekleideten peripheren Darm cin Stückchen in den Rektumstumpf hineinzuzichen und die Ränder des letzteren soviel einzustülpen, daß seine äußere Fläche die Scrosa des crsteren berührt, so wird eine genügende Verklebung der Darmenden schon bald eintreten. Dies gelang mir auch in meinem Falle, wie aus dem Operationsbericht des Näheren nachzusehen ist. Zwar ragte der Dünndarm anfangs portioartig in die Ampulla recti hinein, aber später glich sich dics vollkommen aus.

Viel exakter ließe sich die Vercinigung der Därme in der Weisc ausführen, daß man, wie dic beigelegte Skizze (Fig. 2) 
zeigt, erst durch 3 oder 4 Fäden die Därme ineinander stülpte und sie in dieser Stellung durch Befestigung der Fäden an die Perinealhaut fixierte, um dann die Serosanähte von der Bauchhöhle aus ausführen zu können. Wenn die Fixationsfäden auch nach einigen Tagen durchschneiden, was ja durchaus nicht immer der Fall zu sein braucht, wenn der zuführende Darm nur gehörig mobilisiert worden ist, kann eine Verklebung der Stümpfe wegen der breiten Serosaflächen schon eingetreten sein. Wie ich später aus der Literatur ersehen habe, liegt ein ähnlicher Gedankengang zugrunde für das von $\mathrm{M} \mathrm{ummery}$ angewandte Verfahren zur Wiederherstellung der Kontinuität des Darmrohrs in einem Falle von Exstirpation des unteren Abschnittes der Flexur. Ein in den Stumpf der Flexur eingenähtes Glas- oder Gummidrain wurde von der Bauchhöhle aus ins Rektum eingeführt und vor den Anus gezogen, so daß der Flexurstumpf ein Stückchen ins Rektum invaginiert wurde. Nähte wurden gar nicht angelegt. Am siebenten Tage wurde das Rohr entfernt.

Nachtrag zur Korrektur. Der Patient hat sich zuletzt am I5. Januar I9I2 bei vorzüglicher Gesundheit vorgestellt. Er hat täglich $2-4$ weiche Stühle. Die Übergangsstelle vom Ileumstück ins Rektum hat sich so vollkommen ausgeglichen, daß sie nur als ein ganz dünner, weicher und weiter Narbenring gefühlt werden kann.

\section{Literaturverzeichnis.}

$\therefore$ r. Brunner, "Ein durch Operation geheilter Fall von septischer Thrombose der Mesenterialgefäße. Deutsche Zeitschr. f. Chir., Bd. 89.

2. Deckart, Über Thrombose und Embolie der Mesenterialgefäße. Mitteilg. aus den Grenzgebieten d. Med. u. Chir., Bd. 5, Nr. I8.

3. De $Q$ u e r vain, De l'implantation du colon transverse dans le rectum ou dans l'anus. Revue médicale Igor, Nr. 12. Referiert in Hildebrandts Jahresbericht für I 901, S. 684 .

4. E11i ot. Annals of Surgery 1895, I, S. 9.

5. Il a a $g$ n, Ein durch Operation geheilter Fall von Thrombose im Gebiete der Vena mesenterica superior. Deutsche Zeitschr. f. Chir., Bd. 92.

6. Kölbing, Thrombose der Mesenterialgefäße. Beiträge zur klin. Chir., Bd. 33 .

7. Köster, Zur Kasuistik der Thrombose und Embolie der großen Bauchgefäße. Deutsche med. Wochenschr. I898, Nr. 21. 
8. Krogius, Über cine Nethode das excidierte Rektum durch andere Darmteile mit Vermeidung der Gangrängefahr zu ersetzen. Zentralbl, f. Chir. IgI I, Nr.2I.

9. $\mathrm{K}$ ü $\mathrm{m}$ mell, Rescktion des Colon descendens und Einnähung des Colon transversus in den sphincter ani. Deutscher Chir.-Kongreß 1899.

Io. No nt profit. Un nouvcau procédé de resection du gros intestin. Archiv prov. de Chir. I904, Nr. 3 .

I I. M u m m ery, New method of restoring the continuity of the bovel in cases of excision of a growth low down in the sigmoid flexure. Lancet ig08. May 16.

12. N e utra, Erkrankung der Mesenterialgefäße und ihre Bedeutung für die Chirurgie. Zentralbl. f. d. Grenzgebiete I 902.

13. Sprenge1, Zur Pathologie der Zirkulationsstörungen im Gebiete der Mesenterialgefäße. Archiv f. klin. Chir. 1902. Bd. 67.

14. Vignolo. Referiert von $\mathrm{Giani}$ in Hildebrands Jahresberichte Igos, S. 658 .

I 5. Wil ms. Der Ileus. Dẹutsche Chir., Lieferung 46. 